\title{
Postpartum nutrition
}

\author{
Guidance for general practitioners \\ to support high-quality care
}

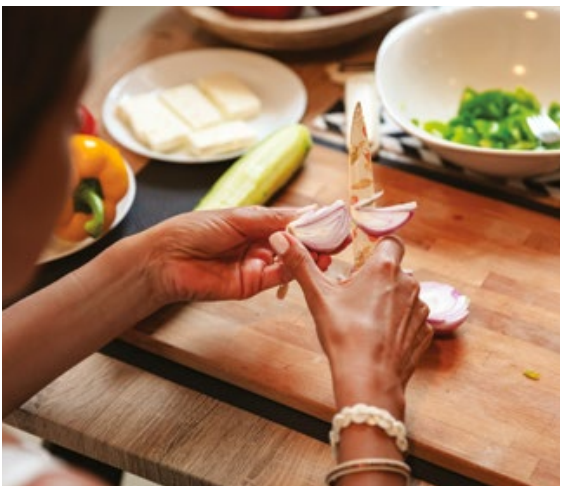

CPD

Lauren Ball, Susan de Jersey,

Joy Parkinson, Lisa Vincze,

Shelley Wilkinson

\section{Background}

General practitioners (GPs) are well placed to support new mothers with evidence-based nutrition care to eat well, live well and care for their new babies. Women who have recently given birth are highly motivated to eat well and seek information and support from GPs.

\section{Objective}

The aim of this article is to review current recommendations on dietary intake for women after birth and how GPs can use this information to inform high-quality ongoing care.

\section{Discussion}

The principles of healthy eating remain the same for all adults, and it is important that GPs support women to consume a wide variety of unprocessed, nutritious foods. Breastfeeding mothers require additional energy and nutrients such as protein, iodine, vitamin B12 and vitamin $D$, which are important in supporting healthy function and infant growth through breastmilk. GPs must take a person-centred approach to consultations, use respectful language and consider practical recommendations to support women in this exciting yet challenging time.
THE POSTPARTUM PERIOD is an exciting yet challenging time, requiring physical, emotional and social adjustment to new norms and expectations for mothers. This period, which includes the immediate six weeks following delivery but can last up to 12 months after childbirth, involves significant bodily changes and learning for mothers, resulting in considerable information seeking for specific health concerns and social support and advice. In addition to recovery from birth, the postpartum period is a critical time within the life course for healthy eating goals to support both a woman's long-term health and future potential pregnancies. Women in Australia often have poor alignment to dietary guidelines during pregnancy, making the postpartum period more critical to address healthy eating goals. ${ }^{1}$ General practitioners (GPs) are well placed to support women with evidence-based care as they strive to eat well, live well and care for their growing families. ${ }^{2}$ GPs often have frequent contact with new mothers, which presents increased opportunities for brief interventions to support lifestyle behaviour change.

\section{Overview of nutrition requirements following pregnancy}

Women who have recently given birth have high metabolic demands as their bodies recover from birth and start to produce breastmilk (if breastfeeding). Women whose babies are exclusively breastfed require approximately $2000 \mathrm{~kJ}$ each day beyond what is recommended for those not breastfeeding, as well as $150 \mu \mathrm{g} /$ day iodine supplementation. Nutrients such as protein, vitamin B12 and vitamin D are also important in supporting healthy function and infant growth through breastmilk (Table 1). GPs are encouraged to check women's antenatal blood tests to identify any signs of micronutrient deficiency, particularly for iron. Additionally, women who are planning another pregnancy should be aware of the additional $400 \mu \mathrm{g} / \mathrm{day}$ of folic acid required one month before and three months after conception for prevention of neural tube defects. There may be an increased risk of birth defects with short interpregnancy intervals if increased nutritional demands are not met. ${ }^{3}$

Despite these increased requirements in key nutrients, the basic principles of healthy eating remain the same as for all adults. Eating a wide variety of foods from the five food groups is encouraged (Table 2).

\section{Weight management following childbirth}

Almost $60 \%$ of women in Australia are overweight or obese; ${ }^{4}$ at eight weeks 
postpartum, this number rises to $63.6 \% .^{5}$ Childbearing has been implicated in the development of obesity, with women retaining an average of $2-3 \mathrm{~kg}$ per pregnancy; ${ }^{6}$ however, there remains wide variability, with one in five women retaining more than $4 \mathrm{~kg}$ at 12 months postpartum. ${ }^{7}$ While the factors associated with this increasing weight trajectory are complex, ${ }^{7}$ a consistent risk factor is gaining more weight than recommended during pregnancy. ${ }^{6,8}$

Supporting women to return to pre-pregnancy weight by six months postpartum, and no later than 12 months postpartum, is associated with more favourable outcomes for future pregnancies and long-term weight trajectory. ${ }^{9,10}$ For those remaining above a healthy weight at this time, actively supporting further weight reduction efforts prior to subsequent pregnancy is recommended. ${ }^{9}$ Women who retain weight at 12 months postpartum are more likely have higher body weight 15 years later. ${ }^{11}$ Irregular and inadequate sleep, disruption to meal routines, lack of access to nutritious foods, physical inactivity and psychosocial considerations such as depression within the postpartum period contribute to postpartum weight retention. ${ }^{7}$ Exclusively breastfeeding to approximately six months postpartum and continuing to breastfeed to 12 months postpartum is positively related to weight reduction, ${ }^{12}$ further supporting the notion of returning to pre-pregnancy weight within this timeframe.

GPs are well placed to provide postpartum weight management care and support for women with interconception care. ${ }^{13}$ However, women report that postpartum visits are often focused on child-related health than targeted to the woman's health. ${ }^{7}$ Supportive, consistent messages to support weight management within GP consultations is feasible. ${ }^{14}$ Capitalising on frequent visits to involve maternal health can potentially affect the lifestyle, weight trajectory and future health risk of mothers and their children.

Referral to other health professionals and programs can be an adjunct to opportunistic brief intervention advice. There is good evidence that postpartum interventions that include interaction with a dietitian and nutrition component, some structured physical activity and weight monitoring support healthy weight attainment. ${ }^{15}$ Importantly, flexibility in delivery modes to help women accommodate challenges experienced in the postpartum period may lead to greater engagement and subsequently better outcomes. ${ }^{15}$

\section{Respectful conversation starters General}

Person-centred care is a key facet of high-quality healthcare by GPs. ${ }^{16}$ In the context of postpartum care, GPs are in a powerful position to influence women's experiences of care based on how key messages are delivered and potentially sensitive topics are raised. Respectful conversations that place the woman at the centre of care are fundamental to ensuring long-term partnerships result in positive experiences for women and their growing families. It is important to ensure that consultations include topics related to her self-care, including diet, nutrition and other lifestyle considerations important to the woman at that time in her life. These conversations can extend from the postpartum period (6-8 weeks) to interconception periods to best prepare for potential future pregnancies.

\section{Weight}

There is now substantial evidence that raising the topic of weight with reproductive women can be challenging for health professionals. ${ }^{17,18}$ Many women, particularly those whose body weight is above the healthy range, have reported stigmatising experiences with health professionals. ${ }^{19,20}$ Despite this challenge, people want and expect to discuss weight with their health providers. ${ }^{19,21}$ The key to entering this dialogue is careful consideration to use non-judgemental and sensitive language. ${ }^{22}$ While it appears that neutral terminology is preferred when raising the issue of weight, such as 'high

\section{Table 1. Requirements of key nutrients for women ${ }^{49}$}

\begin{tabular}{lll}
\hline Nutrient & Requirement if breastfeeding (per day) & Requirement if not breastfeeding (per day) \\
\hline Protein & Age 14-18 years (RDI): $63 \mathrm{~g}(1.11 \mathrm{~g} / \mathrm{kg})$ & Age 14-18 years (RDI): $45 \mathrm{~g}(0.77 \mathrm{~g} / \mathrm{kg})$ \\
& Age 19-50 years (RDI): $67 \mathrm{~g}(1.10 \mathrm{~g} / \mathrm{kg})$ & Age 19-50 years (RDI): $46 \mathrm{~g}(0.75 \mathrm{~g} / \mathrm{kg})$ \\
\hline Calcium & Age 14-18 years (RDI): $1,300 \mathrm{mg}$ & Age 14-18 years (RDI): $1,300 \mathrm{mg}$ \\
& Age 19-50 years (RDI): $1,000 \mathrm{mg}$ & Age 19-50 years (RDI): $1,000 \mathrm{mg}$ \\
\hline lodine & All ages (RDI): $270 \mu \mathrm{g} /$ day & All ages (RDI): $150 \mu \mathrm{g}$ \\
\hline Vitamin B12 & All ages (RDI): $2.8 \mu \mathrm{g} /$ day & All ages (RDI): $2.4 \mu \mathrm{g} / \mathrm{day}$ \\
\hline Water (from fluid) & All ages (RDI): $5.0 \mu \mathrm{g} /$ day $14-18$ years (Al): $2.3 \mathrm{~L}$ fluids & All ages (RDI): $5.0 \mu \mathrm{gg} / \mathrm{day}$ \\
\hline
\end{tabular}


weight' or 'unhealthy weight' instead of 'obese', it is important to acknowledge variation in preferred language. ${ }^{22}$ In practice, this suggests asking each woman what terminology or language she prefers in conversations about weight-related health. ${ }^{23}$ Normalising conversations about weight with all women is likely to reduce negative experiences and promote person-centred care, for example, seeking permission for the discussion by saying 'would it be ok if we discuss your weight today?' followed by a conversation about preferred language. ${ }^{21,22}$

\section{Breastfeeding}

The World Health Organization recommends that all babies are exclusively breastfed until approximately six months of age, then continue to be breastfed supplementarily until two years of age. Breastfeeding protects against child infections, increases intelligence and reduces the likelihood of the child becoming overweight or developing type 2 diabetes later in life. ${ }^{24}$ For mothers, breastfeeding reduces the likelihood of developing breast cancer, ovarian cancer and type 2 diabetes. ${ }^{25}$ Women are less likely to breastfeed when there is not enough breastfeeding information available after birth. ${ }^{26}$ However, GPs may face barriers such as receiving little breastfeeding and nutrition education or support, and may rely mainly on personal experience. $^{27,28}$

Following the birth of a child, women undergo a transformation beyond self; they transition from an individual into a parent. This transition may influence their decision making, particularly for breastfeeding or infant feeding. ${ }^{29}$ Historically, many cultures entrust the responsibility for the care of infants to women. ${ }^{30}$ Over the past four decades, the role structure of couples and families in Western cultures has changed, with more women remaining in the workforce after they become mothers, ${ }^{31}$ and women becoming mothers at an older age. ${ }^{32}$ Partners and grandmothers provide the greatest degree of emotional and practical support for breastfeeding, while GPs and nurses provide information, which is associated with success at establishing lactation. ${ }^{33}$ Reassurance and guidance from GPs can help women to overcome difficulties and find confidence in their own abilities to achieve their feeding goals. ${ }^{34}$ Practical suggestions may support women to meet the increased nutritional requirements for breastfeeding (outlined in Table 2). Box 1 outlines practical suggestions that GPs can provide to women.

\section{Considerations following a pregnancy affected by gestational diabetes}

Of the 300,000 or so Australian women who become pregnant each year, ${ }^{35}$ almost 50,000 will develop gestational diabetes mellitus (GDM), ${ }^{36}$ accelerating their chronic disease trajectory. Of these, almost 8000 will go on to develop type 2 diabetes within 10 years, ${ }^{37}$ and a history of GDM is an independent risk factor for heart disease. ${ }^{38}$

Postnatally, women who have had GDM have been described as falling into a healthcare 'chasm'. ${ }^{39}$ Lack of coordination between hospitals and primary care can result in no one assuming responsibility for ongoing care. Sometimes, GPs may not be aware that a woman has had GDM or have a clear plan for follow-up care. ${ }^{40}$

Australian guidelines recommend that all women who had GDM should undertake a $75 \mathrm{~g}$ oral glucose tolerance test (OGTT) between six and 12 weeks after delivery. ${ }^{41}$ The extent to which these recommendations are integrated into postnatal GP visits is unknown, but some studies suggest diabetes testing is suboptimal. ${ }^{23,42}$ Clinicians report challenges to addressing GDM in Australia, including increasing maternal age, diversity of ethnicity and changes in screening and diagnostic parameters. ${ }^{43}$ It is therefore not surprising that self-report surveys of women with prior GDM indicated that approximately half of participants returned for OGTTs, but only a quarter of these did so in the appropriate period. ${ }^{23,44}$ In the context of a woman being unable to undertake an OGTT postnatally, GPs may discuss alternative options for monitoring glucose status.
GPs are ideally placed to identify women during the postpartum (or interconception) period to play a part in supporting healthy behaviours and weight management. ${ }^{13}$ These strategies contribute to providing a continuum of care before, during and between pregnancies and reducing women's elevated chronic disease risk. Effective approaches to engage women in screening are paramount, as women's motivation to engage in healthy lifestyle behaviours often dramatically reduce once GDM resolves. ${ }^{45}$ Women's lack of perceived risk of developing type 2 diabetes is common and can be related to timing, content and tone of healthcare messages. ${ }^{45,46} \mathrm{~A}$ balance between reassurance of likely resolution of GDM and adequate information about potential progression to type 2 diabetes is required. ${ }^{45}$

Randomised controlled trials have shown that lifestyle modifications with weight loss and moderate exercise can reduce the incidence of type 2 diabetes by up to $58 \%$ for people at high risk. ${ }^{47}$ Real-world implementation in the Australian healthcare system has achieved $40 \%$ reduction in the risk of progression to diabetes. ${ }^{48}$ Unfortunately, few embedded, systematically delivered state or national

\section{Box 1. Practical suggestions to incorporate into clinical practice}

- Consider referral to a lactation consultant to support breastfeeding initiation and continuation.

- Consider advocating for the use of food banks if food insecurity is a factor affecting women's diets.

- Provide information to women on expressing and freezing breastmilk to share feeding opportunities.

- Consider referral to a dietitian for individualised nutrition care.

- Encourage women with practical food suggestions such as using healthy frozen meals that save on preparation time, talking to family and friends to help with healthy food support, or drinking a glass of water during or after each breastfeeding session.

- Suggest small, simple healthy snacks to have on the go, particularly when breastfeeding, such as nuts, seeds, fruit, wholegrain sandwiches, raw vegetables. 
programs exist that acknowledge and address competing demands with a new baby and address issues regarding attending healthcare services. It is important that GPs are aware of local services that may offer flexible approaches to care, including telehealth. Effective programs to support women should meet their articulated needs relating to access, content, delivery style and message. ${ }^{15}$ The program must be underpinned by behaviour change theory and capitalise on the woman's primary focus: her baby. ${ }^{15}$

\section{Conclusion}

GPs are well placed to support new mothers with evidence-based nutrition care to eat well, live well and care for their new babies. Women who have recently given birth are highly motivated to eat well and seek information and support from GPs. The postpartum period is a critical time within the life course for healthy eating goals to support both a woman's long-term health and future potential pregnancies. Women who have recently given birth have high metabolic demands as their bodies recover from birth and start to produce breastmilk (if breastfeeding). Despite these increased requirements in key nutrients, the basic principles of healthy eating remain the same as for all adults. Eating a wide variety of foods from the five food groups is encouraged. A person-centred approach to consultations, using respectful language, and consideration of practical recommendations can support women in this exciting yet challenging time.

\section{Key points}

- GPs are well placed to support mothers with their eating and breastfeeding.

- Breastfeeding mothers need additional energy and iodine to support optimal breastmilk production.

- Protein, vitamin B12 and vitamin D support healthy function and infant growth.

- It is recommended that GPs prioritise supporting women to return to pre-pregnancy weight.

\section{Table 2. Recommended daily intake of core food group serves for women aged 19-50 years ${ }^{24}$}

\begin{tabular}{llll}
\hline Core food group & $\begin{array}{l}\text { Breastfeeding } \\
\text { (serves per day) }\end{array}$ & $\begin{array}{l}\text { Not breastfeeding } \\
\text { (serves per day) }\end{array}$ & Examples of one standard serve \\
\hline $\begin{array}{l}\text { Vegetables and } \\
\text { legumes/beans }\end{array}$ & 5 & $\cdot 1 / 2$ cup cooked vegetables \\
& 7.5 & $\cdot 1 / 2$ cup cooked or canned beans, peas or lentils \\
& & $\cdot 1$ cup green leafy or raw salad vegetables \\
& & $\cdot 1 / 2$ medium potato or other starchy vegetables \\
& & $\cdot 1$ medium tomato \\
\hline
\end{tabular}

\begin{tabular}{lll}
\hline Fruit 2 & 2 & \\
& & 1 medium fruit, such as apple, banana, orange \\
& $\cdot 2$ small fruits, such as apricots, kiwi fruits or plums \\
& $\begin{array}{l}\cdot 30 \mathrm{~g} \text { dried fruit (such as } 4 \text { apricot halves, } 1 \frac{1}{2} \text { tablespoons } \\
\text { sultanas) }\end{array}$
\end{tabular}

Grain (cereal) foods, $\quad 9 \quad 6 \quad \cdot 1$ slice bread, $1 / 2$ medium roll or flat bread (40 $\mathrm{g}$ )

mostly wholegrain and/or

mostly wholegrain and
high-fibre varieties

Lean meats and poultry, fish,

2.5

eggs, tofu, nuts and seeds,

and legumes/beans
- $1 / 2$ cup cooked rice, pasta, noodles, barley, polenta, quinoa

- $1 / 2$ cup cooked porridge, $2 / 3$ cup wheat cereal flakes, $1 / 4$ cup muesli

- 1 crumpet, small English muffin or scone

- $65 \mathrm{~g}$ cooked lean meats, such as beef, lamb, veal, pork (90-100 g raw)

- $80 \mathrm{~g}$ cooked lean poultry, such as chicken, turkey (100 g raw)

- $100 \mathrm{~g}$ cooked fish fillet (115 g raw) or 1 small can of fish

- 2 large eggs

- 1 cup cooked or canned legumes/beans, such as lentils or chickpeas

- 30 g nuts or seeds

\begin{tabular}{lll}
\hline $\begin{array}{l}\text { Milk, yoghurt, cheese and/or } \\
\text { alternatives, mostly reduced fat }\end{array}$ & 2.5 & $2.5 \quad 1$ cup $(250 \mathrm{ml})$ fresh, long-life or reconstituted powdered milk \\
& $\cdot 2$ slices $(40 \mathrm{~g})$ hard cheese, such as cheddar \\
& $\cdot 3 / 4$ cup $(200 \mathrm{~g})$ yoghurt \\
& $\cdot 1$ cup $(250 \mathrm{~mL})$ soy, rice or other cereal drink, with $>100 \mathrm{mg}$ \\
& calcium per $100 \mathrm{~mL}$
\end{tabular}




\section{Authors}

Lauren Ball PhD, AdvAPD, Principal Research Fellow, Menzies Health Institute Queensland and School of Health Sciences and Social Work, Griffith University, Brisbane, Qld

Susan de Jersey PhD, AdvAPD, Clinician Research Fellow, Centre for Clinical Research, Faculty of Medicine, University of Queensland, Brisbane, Qld; Department of Nutrition and Dietetics, Royal Brisbane and Women's Hospital, Metro North Health Brisbane, Qld

Joy Parkinson PhD, Associate Professor, Department of Nutrition and Dietetics, Royal Brisbane and Women's Hospital, Metro North Health, Brisbane, Qld Lisa Vincze PhD, APD, Lecturer, Menzies Health Institute Queensland and School of Health Sciences and Social Work, Griffith University, Brisbane, Old: School of Health Sciences and Social Work, Griffith University, Gold Coast, Qld

Shelley Wilkinson PhD, AdvAPD, Principal Research Fellow, School of Human Movement and Nutrition Sciences, Faculty of Health and Behavioural Sciences, University of Queensland, Brisbane, Qld

Competing interests: $L B$ is a member of the Australian Journal of General Practice Editorial Advisory Committee.

Funding: At the time of publication, LB's salary was supported by a National Health and Medical Research Council fellowship (APP 1173496).

Provenance and peer review: Commissioned, externally peer reviewed.

\section{Correspondence to:}

I.ball@griffith.edu.au

\section{References}

1. Malek L, Umberger W, Makrides M, Zhou SJ. Adherence to the Australian dietary guidelines during pregnancy: Evidence from a national study. Public Health Nutr 2016;19(7):1155-63. doi: 10.1017/S1368980015002232.

2. Ball L, Wilkinson $S$. Nutrition care by general practitioners: Enhancing women's health during and after pregnancy. Aust Fam Physician 2016;45(8):542-47.

3. Petersen JM, Yazdy MM, Getz KD, Anderka MT Werler MM; National Birth Defects Prevention Study. Short interpregnancy intervals and risks for birth defects: Support for the nutritional depletion hypothesis. Am J Clin Nutr 2021;113(6):1688-99. doi: 10.1093/ajcn/nqaa436.

4. Huse O, Hettiarachchi J, Gearon E, Nichols M, Allender S, Peeters A. Obesity in Australia. Obes Res Clin Pract 2018;12(1):29-39. doi: 10.1016/j. orcp.2017.10.002.

5. Fan WQ, Molinaro A. Maternal obesity adversely affects early breastfeeding in a multicultural, multi-socioeconomic Melbourne community. Aust N Z J Obstet Gynaecol 2021;61(1):78-85. doi: 10.1111/ajo.13232.

6. Gunderson EP, Abrams B. Epidemiology of gestational weight gain and body weight changes after pregnancy. Epidemiol Rev 1999;21(2):261-75. doi: 10.1093/oxfordjournals.epirev.a018001.

7. Makama M, Skouteris H, Moran LJ, Lim S. Reducing postpartum weight retention: A review of the implementation challenges of postpartum lifestyle interventions. J Clin Med 2021;10(9):1891. doi: 10.3390/jcm10091891.

8. Rooney BL, Schauberger CW. Excess pregnancy weight gain and long-term obesity: One decade later. Obstet Gynecol 2002;100(2):245-52. doi: 10.1016/s0029-7844(02)02125-7.

9. Villamor E, Cnattingius S. Interpregnancy weight change and risk of adverse pregnancy outcomes: A population-based study. Lancet 2006;368(9542):1164-70. doi: 10.1016/S01406736(06)69473-7.

10. Harrison $\mathrm{CL}$, Teede $\mathrm{H}$, Khan N, et al. Weight management across preconception, pregnancy, and postpartum: A systematic review and quality appraisal of international clinical practice guidelines. Obes Rev 2021;22(10):e13310. doi: 10.1111/obr.13310.

11. Linné $Y$, Dye L, Barkeling B, Rössner S. Longterm weight development in women: A 15-year follow-up of the effects of pregnancy. Obes Res 2004;12(7):1166-78. doi: 10.1038/oby.2004.146.

12. Jiang $M, G a o H$, Vinyes-Pares $G$, et al. Association between breastfeeding duration and postpartum weight retention of lactating mothers: A meta-analysis of cohort studies. Clin Nutr 2018;37(4):1224-31. doi: 10.1016/j.clnu.2017.05.014.

13. Schoenaker DAJM, de Jersey S, Willcox J, Francois ME, Wilkinson S. Prevention of gestational diabetes: The role of dietary intake, physical activity, and weight before, during, and between pregnancies. Semin Reprod Med 2020;38(6):352-65. doi: 10.1055/s-00411723779.

14. Hilder J, Gray L, Stubbe M, Duncan S, Dowell AC. 'Water dripping on a stone': A feasibility study of a healthy weight management conversation approach in routine general practice consultations. Fam Pract 2021;38(3):246-52. doi: 10.1093/ fampra/cmaa122.

15. Vincze L, Rollo M, Hutchesson M, et al. Interventions including a nutrition component aimed at managing gestational weight gain or postpartum weight retention: A systematic review and meta-analysis. JBI Database System Rev Implement Rep 2019:17(3):297-364. doi: 10.11124/ JBISRIR-2017-003593.

16. Brickley B, Williams LT, Morgan M, Ross A Trigger K, Ball L. Putting patients first: Development of a patient advocate and genera practitioner-informed model of patient-centred care. BMC Health Serv Res 2021;21(1):261. doi: 10.1186/s12913-021-06273-y.

17. Heslehurst N, Newham J, Maniatopoulos G, Fleetwood C, Robalino S, Rankin J. Implementation of pregnancy weight management and obesity guidelines: A meta-synthesis of healthcare professionals' barriers and facilitators using the Theoretical Domains Framework. Obes Rev 2014;15(6):462-86. doi: 10.1111/obr.12160.

18. van der Pligt P, Campbell K, Willcox J, Opie J, Denney-Wilson E. Opportunities for primary and secondary prevention of excess gestational weight gain: General practitioners' perspectives. BMC Fam Pract 2011;12:124. doi: 10.1186/1471-2296-12-124.

19. Christenson $A$, Johansson $E$, Reynisdottir $S$, Torgerson J, Hemmingsson E. '....or else I close my ears' How women with obesity want to be approached and treated regarding gestational weight management: A qualitative interview study. PLoS One 2019;14(9):e0222543. doi: 10.1371/ journal.pone.0222543.

20. Incollingo Rodriguez AC, Smieszek SM, Nippert KE, Tomiyama AJ. Pregnant and postpartum women's experiences of weight stigma in healthcare. BMC Pregnancy Childbirth 2020;20(1):499. doi: 10.1186/s12884-020-03202-5.

21. Beeken RJ, Scott AM, Sims R, et al. A Community Jury on initiating weight management conversations in primary care. Health Expect 2021;24(4):1450-58. doi: 10.1111/hex.13286.

22. Puhl RM. What words should we use to talk about weight? A systematic review of quantitative and qualitative studies examining preferences for weight-related terminology. Obes Rev 2020:21(6):e13008. doi: 10.1111/obr.13008.
23. Morrison MK, Collins CE, Lowe JM. Postnatal testing for diabetes in Australian women following gestational diabetes mellitus. Aust N Z J Obstet Gynaecol 2009;49(5):494-98. doi: 10.1111/j.1479828X.2009.01056.x.

24. National Health and Medical Research Council. Australian dietary guidelines. Canberra, ACT: NHMRC, 2013

25. Victora CG, Bahl R, Barros AJ, et al. Breastfeeding in the 21st century: Epidemiology, mechanisms, and lifelong effect. Lancet 2016;387(10017):475-90. doi: 10.1016/S0140-6736(15)01024-7.

26. Gallegos D, Parkinson J, Duane S, Domegan C Jansen E, Russell-Bennett R. Understanding breastfeeding behaviours: A cross-sectional analysis of associated factors in Ireland, the United Kingdom and Australia. Int Breastfeed 2020;15(1):103. doi: 10.1186/s13006-020-00344-2.

27. Holtzman O, Usherwood T. Australian general practitioners' knowledge, attitudes and practices towards breastfeeding. PLoS One 2018;13(2):e0191854. doi: 10.1371/journal. pone.0191854.

28. Crowley J, Ball L, Hiddink GJ. Nutrition in medical education: A systematic review. Lancet Planet Health 2019;3(9):e379-89. doi: 10.1016/S25425196(19)30171-8.

29. Parkinson J, Gallegos D, Russell-Bennett R. Transforming beyond self: Fluidity of parent identity in family decision-making. J Bus Res 2016;69(1):110-19. doi: 10.1016/j. jbusres.2015.07.025.

30. Woodward K. Identity and difference. Thousand Oaks, CA: Sage, 1997.

31. Brough P, Holt J, Bauld R, Biggs A, Ryan C. The ability of work-life balance policies to influence key social/organisational issues. Asia Pac J Hum Resour 2008;46(3):261-74. doi: 10.1177/1038411108095758.

32. Australian Institute of Health and Welfare. Australia's mothers and babies. Canberra, ACT: AIHW, 2021. Available at www.aihw.gov.au/reports/ mothers-babies/australias-mothers-babies/ contents/about [Accessed 28 January 2022]

33. McCarter-Spaulding D, Gore R. Social support improves breastfeeding self-efficacy in a sample of black women. Clin Lactation 2012;3(3):112-15.

34. Fox R, McMullen S, Newburn M. UK women's experiences of breastfeeding and additional breastfeeding support: A qualitative study of Baby Café services. BMC Pregnancy Childbirth 2015;15:147. doi: 10.1186/s12884-015-0581-5.

35. Australian Bureau of Statistics. Births, Australia 2020. Canberra, ACT: ABS, 2020. Available at www.abs.gov.au/statistics/people/population/ births-australia/latest-release [Accessed 28 January 2022].

36. Australian Institute of Health and Welfare. Incidence of gestational diabetes in Australia 2019. Cat. no. CVD 85. Canberra, ACT: AIHW, 2019. Available at www.aihw.gov.au/reports/diabetes/ incidence-of-gestational-diabetes-in-australia/ contents/changing-trends [Accessed 28 January 2022].

37. Lee AJ, Hiscock RJ, Wein P, Walker SP, Permezel M. Gestational diabetes mellitus: Clinical predictors and long-term risk of developing type 2 diabetes: A retrospective cohort study using survival analysis. Diabetes Care 2007:30(4):878-83. doi: 10.2337/dc06-1816.

38. Gunderson EP, Sun B, Catov JM, et al. Gestational diabetes history and glucose tolerance after pregnancy associated with coronary artery calcium in women during midlife: The CARDIA study. Circulation 2021;143(10):974-87. doi: 10.1161/ CIRCULATIONAHA.120.047320. 
39. Institute of Medicine (US) Committee on Quality of Health Care in America. Crossing the quality chasm: A new health system for the 21st century. Washington, DC: National Academies Press (US) 2001.

40. Wilkinson SA, Lim SS, Upham S, et al. Who's responsible for the care of women during and after a pregnancy affected by gestational diabetes? Med J Aust 2014;201(3 Suppl):S78-81. doi: 10.5694/mja14.00251.

41. Nankervis A, Mclntyre HD, Moses RG, Ross GP, Callaway LK. Testing for gestational diabetes mellitus in Australia. Diabetes Care 2013:36(5):e64. doi: 10.2337/dc12-2345.

42. Wilkinson SA, Brodribb WE, Upham S, Janamian T, Nicholson C, Jackson CL. Primary care of women after gestational diabetes mellitus: Mapping the evidence-practice gap. Med J Aust 2014;201(3 Suppl):S74-77. doi: 10.5694/ mja14.00264.

43. Laurie JG, Mclntyre HD. A review of the current status of gestational diabetes mellitus in Australia - The clinical impact of changing population demographics and diagnostic criteria on prevalence. Int J Environ Res Public Health 2020;17(24):9387. doi: 10.3390/ijerph17249387.

44. Sterne V, Logan T, Palmer M. Factors affecting attendance at postpartum diabetes screening in women with gestational diabetes mellitus. Pract Diabetes 2011;28(2):64-68a. doi: 10.1002/pdi.1559.

45. Lie ML, Hayes L, Lewis-Barned NJ, May C, White M, Bell R. Preventing type 2 diabetes after gestational diabetes: Women's experiences and implications for diabetes prevention interventions. Diabet Med 2013;30(8):986-93. doi: 10.1111/ dme.12206.

46. Kim C, McEwen LN, Piette JD, Goewey J, Ferrara A Walker EA. Risk perception for diabetes among women with histories of gestational diabetes mellitus. Diabetes Care 2007;30(9):2281-86. doi: 10.2337/dc07-0618.

47. Keely E, Clark H, Karovitch A, Graham I. Screening for type 2 diabetes following gestational diabetes: Family physician and patient perspectives. Can Fam Physician 2010;56(6):558-63.

48. Dunbar JA, Jayawardena A, Johnson G, et al. Scaling up diabetes prevention in Victoria, Australia: Policy development, implementation, and evaluation. Diabetes Care 2014;37(4):934-42. doi: $10.2337 /$ dc12-2647.

49. National Health and Medical Research Council. Nutrient reference values for Australia and New Zealand. Canberra, ACT: NHMRC, 2006. 